\title{
Andreas Losch \\ „Der Ewige“ als „Synthese“ des Stern. Der Gebrauch des Gottesnamens „der Ewige“ bei Franz Rosenzweig
}

\begin{abstract}
In his famous final essay Franz Rosenzweig neglects to mention that he had referred to the expression "the Eternal" already in The Star of Redemption. Through a comprehensive analysis of his writings, and in particular of his masterpiece, the article seeks to research thoroughly Rosenzweig's use of this expression. The revelation of God's name is a core issue in The Star of Redemption. While elaborating on the structure of the Star, Rosenzweig seeks to capture the crucial meaning of God's name by translating it as "the Eternal," thereby enabling us to consider the thought of eternity as presenting the "synthesis" within the system of The Star of Redemption. Commonly perceived to convey an infinite progression of time, Rozenzweig's understanding of the concept of eternity in The Star proves to be richer, including the idea of the everlasting as well as the notion of a constantly renewing presence. The article furthermore shows that the concept of the meaning of God's name oriented at the present, as devised by Rosenzweig together with Martin Buber in their joint work of translation, had already been articulated in The Star.
\end{abstract}

DOI 10.1515/naha-2015-0008

Bekanntermaßen ist „Der Ewige“ Titel von Rosenzweigs berühmtem letztem Aufsatz. Darin macht Rosenzweig allerdings nicht deutlich, dass er selbst den „Ewigen“ als Gottesnamen im Stern der Erlösung zentral verwendet. ${ }^{1}$ Der vorliegende Artikel möchte von dieser Beobachtung aus dem Gebrauch des Ausdrucks „der Ewige“ als Gottesname in Rosenzweigs Schriften nachspüren, und zwar vor allem im Stern, wo Rosenzweig diese Wiedergabe des Gottesnamens fast durchweg verwendet. Insbesondere ist der Frage nachzugehen, ob die als Zentralstelle des Stern der Erlösung identifizierte Stelle der Namensoffenbarung dort nicht nur allgemein den Gottesnamen, sondern diesen in einer ganz bestimmten Übersetzung und Sinnkonnotation meint, nämlich als „Ewigen“. Um

1 Diesen Hinweis verdanke ich Paul Mendes-Flohr, dem ich diesen Aufsatz widmen möchte.

Andreas Losch: University of Bern (Switzerland), Center for Space and Habitability, c/o Theological Faculty, Länggassstr. 51, CH-3012, Bern, E-Mail: andreas.losch@csh.unibe.ch 
diese Stelle zu verstehen, ist zu klären, wie Rosenzweig den Begriff „ewig“ interpretiert.

„Der Ewige“ als Gottesname ist nichts Geringeres als die „Synthese“ des Stern (im Rosenzweig'schen Sinne des Wortes ${ }^{2}$ ), so die hier zu entfaltende These. ${ }^{3}$ Der Aufsatz „Der Ewige“ lässt sich von daher als Kommentar zu Rosenzweigs eigener Entwicklung im Umgang mit dem Gottesnamen lesen. ${ }^{4}$

Beginnen wir mit der Untersuchung des Gebrauchs des Gottesnamens im Stern der Erlösung. Auch wenn in Teil III „Der Herr“ einmal als Überschrift erscheint, ${ }^{5}$ wird doch in Bibelzitaten im Stern von Rosenzweig sonst immer „der Ewige“ als Wiedergabe des Gottesnamens gewählt. ${ }^{6}$ Dies ist umso bedeutsamer, als in den darauf folgenden Werken, auch in der Übersetzung von Werken des Jehuda Ha-Levi, die Wiedergabe des Gottesnamens zwar schwankend ist, aber nie mehr „der Ewige“ benutzt wird. ${ }^{7}$ Es ist daher anzunehmen, ${ }^{8}$ dass der Gebrauch dieser Wiedergabe des Gottesnamens im Stern umso bestimmtere Bedeutung hat.

2 Rosenzweig unterscheidet „unsre Synthesis“ von der idealistischen Synthesis. Vgl. Franz Rosenzweig, Der Stern der Erlösung, in: Franz Rosenzweig, Der Mensch und sein Werk. Gesammelte Schriften (im Folgenden GS) Bd. 2, hrsg. von Reinhold Mayer (Den Haag: Nijhoff, 1976), 256.

3 Ähnlich hat bereits Renate Schindler beobachtet: „Der Ausgangspunkt von Rosenzweigs Kritik an der europäischen Philosophiegeschichte und am idealistischen Denken in der Form, die Hegel ihm gegeben hat, ist der Begriff von Ewigkeit, die mit der zeitlichen Dimension einer AllGegenwart gleichgesetzt wird.“ Renate Schindler, Zeit, Geschichte, Ewigkeit in Franz Rosenzweigs Stern der Erlösung (Berlin: Parerga, 2007), 20.

4 Diese Behauptung wird im Zuge der hier unternommenen Argumentation zu beweisen sein. Sie ließe sich natürlich wunderbar überprüfen, wenn von Nahum Glatzer, der für Rosenzweig die Recherchen zum Aufsatz „Der Ewige“ durchführte, diesbezügliche Notizen überliefert wären. Leider befinden sich in dem Glatzer-Archiv an der Vanderbilt University Nashville zwar die umfangreichen Rechercheergebnisse, die Glatzer Rosenzweig zugänglich machte (Box \#3/215), aber kein Kommentar zur Aufgabenstellung selbst.

5 Rosenzweig, Stern der Erlösung, 427. Dazu später mehr. Hinzu kommt die im Übergang von Teil I auf Teil II erfolgende Erwähnung des „Welttags des Herrn“. Rosenzweig, Stern der Erlösung, 95. Siehe dazu den Abschnitt unten „Am Ende das Schweigen“.

6 Rosenzweig, Stern der Erlösung, 196, 198, 281, 303, 356, 442, 471. Die ältere englischsprachige Übersetzung von William Hallo (Franz Rosenzweig, The Star of Redemption, Notre Dame, IN: University of Notre Dame Press, 1985) gibt dies allerdings nicht wieder, erst die neuere Übersetzung von Barbara Galli (Franz Rosenzweig, The Star of Redemption, Madison, WI: University of Wisconsin Press, 2005) gibt darauf acht.

7 So die Auskunft von Nadine Schmahl, Das Tetragramm als Sprachfigur (Tübingen: Mohr, 2009), $120 \mathrm{ff}$.

8 Gegen Schmahl, Tetragramm, 75-76. 
Wieso aber wählt Rosenzweig im Stern fast durchgängig „der Ewige“ als Wiedergabe, und wie kommt er darauf, gerade diese Wiedergabe zu benutzen? Schließlich, wieso gibt er diese Übersetzung danach auf?

Offensichtlich war ihm diese von Moses Mendelssohn gewählte Übertragung des Gottesnamens ${ }^{9}$ von Jugend auf bekannt. In einem Tagebucheintrag vom 29.9.1906, Rosenzweig war damals neunzehn Jahre alt, benutzt er „der Ewige" ganz selbstverständlich, ${ }^{10}$ und es wird aus zwei frühen Briefen deutlich, dass Rosenzweig natürlich auch mit Mendelssohn vertraut war. ${ }^{11}$

Aus einem späteren Brief ${ }^{12}$ geht zudem hervor, dass Rosenzweig in der Zeit nach dem Verfassen des Stern zusammen mit Ernst Cassirer und Max Hermann für die Leitung oder sogar für die Oberleitung ${ }^{13}$ einer Mendelssohnausgabe angefragt worden war, wobei er auch darüber nachdachte, konkrete Mitarbeit zu übernehmen. ${ }^{14}$ Allerdings wird auch deutlich, dass für ihn dabei noch eine gewisse Einarbeitung in die Materie vonnöten gewesen wäre. Er schreibt im Juni 1919, er „brauche mindestens 14 Tage, wenn nicht 4 Wochen, um ,Mendelssohnkenner' zu werden". ${ }^{15}$ Das Projekt kam jedoch offensichtlich nicht zustande.

Die Lektüre von Rosenzweigs früheren Briefen und Tagebüchern jedenfalls kommt über die damals übliche Vertrautheit mit der Mendelssohn'schen Wiedergabe hinaus zu dem Schluss, dass Rosenzweig, wie es sehr viel später ja auch im Aufsatz „Der Ewige“ thematisiert wird, ${ }^{16}$ die Übersetzung „Herr“ aufgrund ihres christlichen Gebrauchs problematisch erscheint. Es ist gerade der Brief an Rudolf Ehrenberg, welcher Rosenzweigs Begründung für seine Revision der Entscheidung des Leipziger Nachtgesprächs enthält („Ich bleibe also Jude“), in dem er den Gebrauch von „Herr“ kritisch als spezifisch christlich markiert. ${ }^{17}$ So benutzt Rosenzweig diese Wiedergabe im Folgenden eigentlich nur in der

9 Vgl. dazu Franz Rosenzweig, „Der Ewige“, in: Martin Buber/Franz Rosenzweig, Die Schrift und ihre Verdeutschung (Berlin: Schocken, 1936), 184-210. Siehe dazu auch Rivka Horwitz, „Moses Mendelssohns Interpretation des Tetragrammaton: ,Der Ewige““, in: Judaica 55 (1999), 2-19; 132-152.

10 Franz Rosenzweig, GS Bd.1, Briefe und Tagebücher, 1. Band 1900-1918, hrsg. von Rachel Rosenzweig/Edith Rosenzweig-Scheinmann (Den Haag: Nijhoff, 1979), 57.

11 Rosenzweig, Briefe und Tagebücher 1, 62, 80.

12 Franz Rosenzweig, Brief an Margrit Rosenstock vom 4.6.1919, The Gritli Letters (Gritli Briefe), http://argobooks.org/gritli/index.html [23.9.2014].

13 Rosenzweig, Brief an Margrit Rosenstock vom 25. und 26.6.1919.

14 Rosenzweig, Brief an Margrit Rosenstock vom 27.6.1919.

15 Rosenzweig, Brief an Margrit Rosenstock vom 25. und 26.6.1919.

16 Rosenzweig, Der Ewige, 188, 196.

17 Rosenzweig, Briefe und Tagebücher 1, 134-135, 137. 
wortgetreuen Wiedergabe christlicher Bibelzitate, ${ }^{18}$ während er in seinen Tagebucheinträgen hebräisch schreibt und also gar nicht übersetzt. ${ }^{19}$

Den Ausdruck „der Ewige“ benutzt Rosenzweig wieder im Dezember 1917 in einem Brief an Richard Ehrenberg ${ }^{20}$ und zitiert in einem Brief an Margrit Rosenstock auch Cohen mit einer Passage, in der Cohen diese Wiedergabe des Gottesnamens benutzt. ${ }^{21}$

Ergebnis der Brieflektüre ist also bis zum Erscheinen des Stern der Erlösung eine natürliche Vertrautheit mit der Mendelssohn'schen Wiedergabe, die sich damit auch als spezifisch jüdische Wiedergabe des Gottesnamens für den Stern der Erlösung anbietet. Rosenzweigs eigene Analyse nach späterer intensiverer Beschäftigung mit Mendelssohn hält fest: Der Gottesname „der Ewige“ „ist nicht bloß von den meisten späteren [d.h. nach-Mendelssohn'schen] jüdischen Bibelübersetzungen übernommen worden, sondern auch in die gebräuchlichen Gebetsübersetzungen, in die Predigten und in alles sonst noch im Kult und im Zusammenhang mit dem Kult deutsch Gesprochene eingedrungen und hat so die jüdische Frömmigkeit des Emanzipationszeitalters überall, selbst bis in die Kreise der Orthodoxie hinein [...] gefärbt.“22 Und das gilt auch für Rosenzweig selbst.

\section{Die Offenbarung des Gottesnamens als Orientierung}

Im Folgenden soll zunächst die bereits von Margarete Susman ${ }^{23}$ als solche identifizierte Zentralstelle des Stern der Erlösung diskutiert werden, in der es um die Offenbarung des Gottesnamens geht. Erst nach der Darstellung der Entwicklung dieses Gedankens soll gefragt werden, ob dabei an einen bestimmten Gottesnamen wie „der Ewige“ zu denken ist.

18 Rosenzweig, Briefe und Tagebücher 1, 167, 264.

19 Rosenzweig, Briefe und Tagebücher 1, 157-159, 169. Es ist im Übrigen erwähnenswert, dass die von den Herausgebern der Briefe und Tagebücher bei Bibelzitaten immer hinzugefügte Buber/Rosenzweig-Übersetzung mit ihrer spezifischen Wiedergabe des Gottesnamens durch das Personalpronomen in Kapitälchen diese Entwicklung und Positionierung bei Rosenzweig durchaus verschleiert.

20 Rosenzweig, Briefe und Tagebücher 1, 503.

21 Rosenzweig, Brief an Margrit Rosenstock vom 23.4.1918.

22 Rosenzweig, Der Ewige, 186.

23 Margarete Susman, „Der Stern der Erlösung“, in: Der Jude. Eine Monatsschrift 6 (1921/22), 259-264, und Franz Rosenzweig, GS Bd. 1, Briefe und Tagebücher, 2. Band: 1918-1929, 752, 758. 
Dass die erwähnte Passage im Stern von zentraler Bedeutung ist, wird auch daran deutlich, dass hier Rosenzweigs Offenbarungsbegriff zutage tritt. Dieser ist aber nun keine vollkommen eigenständige Erfindung Rosenzweigs, sondern wird im Dialog mit Eugen Rosenstock gewonnen. Rosenstock antwortet Rosenzweig in einem Brief vom 28.10.1916 auf dessen Frage nach seinem ,jetzigen Begriff vom Verhältnis von Natur und Offenbarung “. ${ }^{24}$ Rosenzweigs Frage selbst ist möglicherweise eine Reminiszenz an das Leipziger Nachtgespräch, ${ }^{25}$ denn mit der Erinnerung daran hatte Rosenzweig den Brief begonnen, in dem er jene Frage stellte. Rosenstocks Antwort nun lautet:

„Die Offenbarung bedeutet den Anschluß auch unseres Bewußtseins an den über die Erde hinausreichenden Welt- und Himmelszusammenhang. Die Frage, die Sie stellen: ,Natur und Offenbarung“ kann ich nur verstehen als ,natürlicher Verstand und Offenbarung‘. Natur und Offenbarung sind ja nicht vergleichbar. Der natürliche Verstand also kennt vorn und hinten, links und rechts, und behilft sich in diesem Geviert mit einem Netz von Analogien. Er vergleicht und hinkt so in der ungeheuren Weite von einem Platz zum nächsten fort. [...] Der Entschluß, in diesem Raumgeviert die eigene Position nicht als erkennende Mitte, sondern als von oben bedingt anzunehmen, dieser Verzicht,

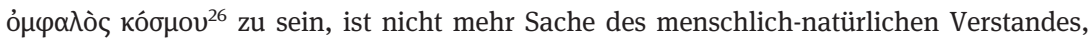
sondern ist jenes Vermögen in uns, das Offenbarung an uns, in uns und für uns möglich macht.“

Ganz ähnlich klingt es dann im Brief Rosenzweigs an Gertrud Oppenheim vom 30.5.1917, wo dieses Rosenstock'sche Offenbarungskonzept um die Dimension der Zeit erweitert und mit der Bedeutung des Namens verknüpft wird:

„Denn die Offenbarung begründet ein Oben und Unten, ein Europa und Asien usw. einerseits und ein Früher und Später, Vergangenheit und Zukunft andererseits. Das Grenzenlose (,Absolute!) steigt zur Erde nieder und zieht von hier aus, von dem Orte seines Niedersteigens, Grenzen in das Meer des Raums und die Strömung der Zeit. Das ists. Oben und unten, trotz Kopernikus. Nord, Süd, Ost und West, statt daß ich mich bloß herumzudrehen brauche, um aus rechts links zu machen. Vergangenes und Zukünftiges, statt daß ich bloß ein bißchen später oder früher zu leben brauche und andres ist vergangen, andres zukünftig. [...] Statt des Überall des unnennbaren Gefühls das ,den Finger drauf!' des Namens. Und zwar des Namens, der nicht ,Schall und Rauch' ist. Nicht Schall und Rauch, sondern Wort und Feuer. Wo das Wort gehört wird, da ist es vorbei

24 Franz Rosenzweig, Briefe. Ausgewählt und hrsg. von Edith Rosenzweig (Berlin: Schocken, 1935), 675.

25 Zum Leipziger Nachtgespräch siehe z. B. Dietmar Kamper, „Das Nachtgespräch vom 7. Juli 1913. Eugen Rosenstock-Huessy und Franz Rosenzweig“, in: Wolfdietrich Schmied-Kowarzik, Der Philosoph Franz Rosenzweig (1886-1929). Bd. I: Die Herausforderung jüdischen Lernens (Freiburg im Breisgau: Alber, 1988), 97-104.

26 „Nabel der Welt“. 
mit dem Schweigen, der Stille, der Stummheit, und auch dem Lärm, dem Schrei, dem Tierlaut. Wo das Feuer brennt, da gibt es keine Kälte und kein Dunkel. Es gibt zwar noch all das, aber eben nur dort wo das Wort und das Feuer noch nicht hingedrungen ist; aber es sind ihnen ja keine Grenzen gesetzt; das Wort tönt fort durch die Zeit, von Mund zu Mund, und das Feuer breitet sich aus in dem Raum.“27

Hier findet sich sicher eine Anspielung auf den brennenden Dornbusch. Doch auch das zentrale Bild des Stern der Erlösung, das Kernfeuer des Sterns, von dem die Strahlen in die Nacht schießen, scheint hier bereits vorgeformt. In einem anschließenden Brief an Gertrud Oppenheim vom 31.5.1917 ${ }^{28}$ wird erneut die Verbindung des Konzepts mit dem Offenbarungsbegriff (und der Bezug auf Ex 3,14) deutlich:

„Es kommt alles darauf an, daß man den Namen nicht bloß als Schall und Rauch, also nicht bloß als Nach- und Nebenbild der Dinge erkennt, sondern, ganz kraß gesagt, als ein Ding unter Dingen, Wort neben Worten, Feuer neben Feuern. Gott ist nicht alles, sondern ,von ihm und $z u \mathrm{ihm}^{\prime}$ ist alles. Also gelehrt ausgedrückt: er steht zu allem in Beziehung. Er ist nur einiges, genau gesagt sogar nur eines, der Punkt des Herniedersteigens, der Horizont in dem sich Himmel und Erde berühren."29

Auf systematischer Ebene wird an dieser Stelle bereits die spätere Stellung Gottes im Stern deutlich, der dort die Spitzenposition im ursprünglichen Dreieck einnimmt. Man vergleiche dazu die sogenannte Urzelle des Sterns, den Brief Rosenzweigs an Rudolf Ehrenberg vom 18.11.1917. Auch dort macht Rosenzweig deutlich, dass der gefundene Offenbarungsbegriff sein „philosophischer Archimedespunkt“30 ist. Dennoch war ihm Rosenstocks Deutung „noch nicht genug““. ${ }^{31}$ Neu ist allerdings, so Rosenzweig, nur das (im Weizsäcker'schen Sinne) „Systematische“ seiner Deutung. ${ }^{32}$

„[...] Erst von hier [der Mitte; A. L.] aus gesehen werden auch Anfang und Ende aus Grenzbegriffen der Unendlichkeit zu Eckpfeilern unsres Weltbesitzes, der ,Anfang` zur Schöpfung, das ,Ende‘ zur Erlösung. Die Offenbarung also ist fähig, Mittelpunkt zu sein, fester unverrückbarer Mittelpunkt. Und warum? Weil sie dem Punkt geschieht, dem starren tauben und unverschiebbaren Punkt, dem trotzigen Ich, das ,ich nun einmal bin““, ${ }^{33}$

27 Rosenzweig, Briefe und Tagebücher 1, 413.

28 Rosenzweig, Briefe und Tagebücher 1, 414.

29 Rosenzweig, Briefe und Tagebücher 1, 414.

30 Franz Rosenzweig, ,,Urzelle“ des Stern der Erlösung“, in: Franz Rosenzweig, GS Bd. 3, Zweistromland. Kleinere Schriften zu Glauben und Denken, hrsg. von Reinhold Mayer/Annemarie Mayer (Dordrecht/Boston/Lancaster: Nijhoff, 1984), 125-138, 125.

31 Rosenzweig, „Urzelle“, 126.

32 Rosenzweig, „Urzelle“, 126.

33 Rosenzweig, „Urzelle“, 133. 
womit Rosenzweig in der „Urzelle“ die linke Seite seines Ursprungsdreiecks beschreibt, ${ }^{34}$ welches auch in der Figur des Stern noch die Offenbarung im engeren Sinn kennzeichnet. ${ }^{35}$ Im Text des Stern heißt es dazu so:

„Was einen eigenen Namen hat [...], ist seine eigene Gattung. Es hat auch nicht mehr seinen Ort in der Welt, seinen Augenblick im Geschehen, sondern es trägt sein Hier und Jetzt mit sich herum; wo es ist, ist ein Mittelpunkt, und wo es den Mund öffnet, ist ein Anfang.“36

Das Ich mit seinem Eigennamen verlangt nun nach Orientierung. Den „Mittelpunkt im Raum, [...] Anfang in der Zeit“37 bietet der göttliche Name als „Grund der Offenbarung“.

„Denn wahrhaftig, Name ist nicht, wie der Unglaube immer wieder in stolz-verstockter Leere wahrhaben möchte, Schall und Rauch, sondern Wort und Feuer. Den Namen gilt es zu nennen und zu bekennen: Ich glaub ihn." ${ }^{\text {38 }}$

Dies schrieb Rosenzweig mit eigenen Worten „damals im November 18 mit dem ganz klaren Bewußtsein, daß es der Kern- und Mittelsatz des Ganzen sei“. ${ }^{39}$ Die

34 Vgl. folgende Abbildung, entnommen aus Rosenzweig, „Urzelle“, 136:

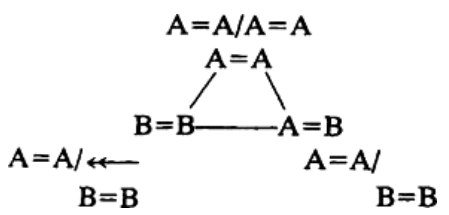

Dabei ist, wie Rosenzweig im Stern noch deutlicher macht, A=A Symbol für Gott, B=B Symbol für den Menschen und A=B (bzw. im Stern dann B=A) Symbol für die Welt.

35 Vgl. Andreas Losch, „Der Stern, im Licht der Offenbarung betrachtet - zur geometrischen Konstruktion des Sterns der Erlösung“, in: Naharaim - Zeitschrift für deutsch-jüdische Literatur und Kulturgeschichte 5 (2011). These dieses früheren Aufsatzes ist es, „dass man die Gestalt des Sterns der Erlösung en détail in geometrischer Form darstellen kann, so dass daraus die Urzelle und eben auch der zum Symbol gewählte Davidsstern ersteht“ (Losch, Geometrische Konstruktion, 36). Diese These, dass es einen solchen Stern als Zeichnung gibt (und ggf. auch materialiter gegeben haben muss), lässt sich u. a. aus folgendem Rosenzweig-Zitat belegen: „Den Stern selbst werde ich wahrscheinlich nur im Übergang von II zu III ganz kurz dem ,Leser 'zur Selbstkonstruktion empfehlen, im übrigen einfach die Symbole $\Delta \nabla$ auf ein Blatt vor je den I.II.III. Teil malen. Im übrigen muss alles mit Worten zu sagen sein.“ Rosenzweig, Brief an Margrit Rosenstock vom 19.10.1918. Der Stern erschöpft sich also nicht in der Kombination der Dreieckssymbole, sondern ist vermutlich wesentlich detaillierter als diese groben Symbole.

Die unteren beiden Ecken des Sterns werden übrigens aufgrund der unklaren Beschreibung in der "Schwelle“ oft vertauscht, allerdings ist dies keinesfalls gleichgültig.

36 Rosenzweig, Stern der Erlösung, 208.

37 Rosenzweig, Stern der Erlösung, 209.

38 Rosenzweig, Stern der Erlösung, 209.

39 Rosenzweig, Briefe und Tagebücher 2, 752, 758. 
Bedeutung des Namens für Rosenzweig, insbesondere des offenbarten Gottesnamens, wird hier offensichtlich. Er ist das Feuer, der Busch und Stern brennen lässt, wie er in die Nacht strahlt.

Ich möchte im Folgenden darlegen, ${ }^{40}$ dass Rosenzweig sich im Stern nun gewissermaßen „selbst beim Wort nimmt“ und diese Zentralstellung des offenbarten Gottesnamens in dem im Stern der Erlösung entwickelten System auch einlöst, und zwar im Wesentlichen anhand der Wiedergabe des offenbarten Namens, wie sie ihm von Jugend an vertraut war: dem Wort „der Ewige“. Ich meine also nicht, dass „der Ewige“ als Gottesname bereits im Blick war, als Rosenzweig über Offenbarung und Orientierung und die Bedeutung des Gottesnamens nachgedacht hat. Ich versuche vielmehr zu zeigen, dass er in der Konstruktion des Stern diese Gedanken dann auf den ihm vertrauten Gottesnamen angewandt und so System und Offenbarung zusammengebracht hat.

Angesichts der Vorgeschichte von Rosenzweigs Abgrenzung vom christlich benutzten „Herr“ wird mit dem durchgängigen Gebrauch von „der Ewige“ auch deutlich, dass der Stern der Erlösung ein durch und durch jüdisches Buch ist. ${ }^{41}$ So versteht man im Übrigen auch den „Schrecken“, der Rosenzweig widerfährt, als er später bei der Vorbereitung des Aufsatzes „Der Ewige“ erfährt, dass dieser Gottesname keineswegs exklusiv jüdisch gebraucht wird, sondern „in französisch reformierten Bibeln des 19. Jahrhunderts der Gottesname durchweg mit l'Éternel wiedergegeben wird“. ${ }^{42}$

\section{„Der Ewige“ als „Synthese“ des Stern}

Welche Rolle spielt nun der Gottesname „der Ewige“ im Stern der Erlösung? Dazu wollen wir zunächst einen Blick auf sein erstes Vorkommen werfen.

Erwartungsgemäß taucht „der Ewige“ als Gottesname zuerst im zentralen Offenbarungsbuch (II/2) auf, im Schriftzitat des „Gebots aller Gebote“: „Du sollst lieben den Ewigen, deinen Gott, von ganzem Herzen und von ganzer Seele und aus allem Vermögen." 43 Vorher wird der Gottesname nicht verwendet und es gibt zuvor auch kein Schriftzitat mit Erwähnung des Gottesnamens, in dem dies erforderlich wäre.

40 Was bisher, auch von Anna Elisabeth Bauer, übersehen worden ist. Anna Elisabeth Bauer, Rosenzweigs Sprachdenken im „Stern der Erlösung“ und in seiner Korrespondenz mit Martin Buber zur Verdeutschung der Schrift (Frankfurt am Main/New York: Lang, 1992).

41 Vgl. allerdings die Differenzierung unten im Abschnitt „Am Ende das Schweigen“.

42 Rosenzweig, Briefe und Tagebücher 2, 1220.

43 Rosenzweig, Stern der Erlösung, 196. 
Was verrät uns diese Stelle über die Verwendung des Gottesnamens? Der Kontext handelt von der „Grammatik des Eros“, der „Sprache der Liebe“, ${ }^{44}$ die im Modus des Imperativs eingeordnet wird und von daher Gebotsform hat. Dies unterscheidet die Sprachgestalt der Offenbarung von derjenigen der Schöpfung und der Erlösung, wobei die Verwendung dieser drei Kategorien selbst bereits auch eine gewisse Kenntnis der Offenbarung deutlich macht. In der Schöpfung, die Rosenzweig dem Modus des Indikativs zuordnet, ist das Stammwort das bejahende „gut“, wie es sich ja bekanntlich im Schöpfungsbericht wiederholt. Das Stammwort der Offenbarung lautet nun „Ich“ als „laut gewordenes Nein“.45 Zwei Dinge sind an dieser Stelle zu klären: ${ }^{46}$ 1) Warum wird hier ein „Nein“ laut? 2) Warum gilt das „Ich“ als „Nein“?

1) Man denke bei der ersten Frage an die Systematik des Stern: ${ }^{47}$ In jedem der drei Elemente Gott, Welt und Mensch findet ein Prozess der Selbstschöpfung, Selbstoffenbarung und Selbsterlösung unter den Stammworten Ja, Nein und Und statt. Treten diese Elemente nach außen und werden so wirk-lich, wiederholt sich dieser Prozess in dem Dreieck von Gott, Welt und Mensch. Und wieder steht jeweils - wie auch in der Endgestalt des Sterns - die Schöpfung unter dem Ja, die Offenbarung unter dem Nein und die Erlösung unter dem Und. ${ }^{48}$ So weit die systematische Betrachtung.

44 Kapitelüberschrift (Nr. 154) in Rosenzweig, Stern der Erlösung, 193.

45 Rosenzweig, Stern der Erlösung, 193.

46 Ich beschränke mich hier auf die Erörterung des Kontextes. Für eine philosophiegeschichtliche Einordnung siehe Hans Martin Dober, Die Zeit ernst nehmen. Studien zu Franz Rosenzweigs „Der Stern der Erlösung“ (Würzburg: Königshausen \& Neumann, 1990), 192f., 209-211.

47 Vgl. Losch, Geometrische Konstruktion.

48 Losch, Geometrische Konstruktion, 44, verdeutlicht diese Systematik, die hier nur skizzenhaft wiedergegeben werden kann:

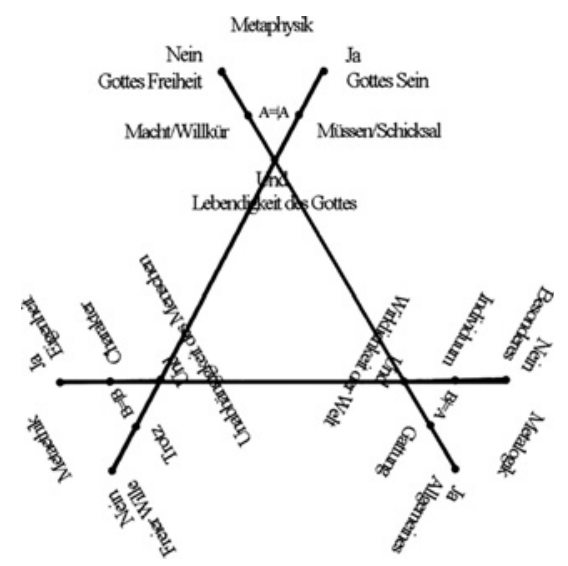


2) Warum aber gilt gerade das „Ich“ als Stammwort der Offenbarung und als „Nein“? Allgemein drückt das Ich die Individuation eines Subjekts aus. ${ }^{49}$ Rosenzweig antwortet so: „Ich ist stets ein laut gewordenes Nein. Mit „Ich“ ist immer ein Gegensatz aufgestellt, es ist stets unterstrichen, stets betont; es ist immer ein ,Ich aber" " ${ }^{\circ 0}$ Er gewinnt so allerdings einen Begriff von einem Ich, „das vom Ich des Idealismus dadurch unterschieden ist, daß es nicht in einer monologischen Bewegung der Reflexion sich setzt, indem es sich vom Nichtich abgrenzt, sondern das sich setzt, indem es sich ausspricht“".51 Biblischen Beleg für die Verbindung des Ich mit der Offenbarung findet Rosenzweig sodann im Anfang des Dekalogs, im "אנכי יהוה אלהיך" (Ex 20,2), wo Rosenzweig ebenfalls den Gottesnamen mit „der Ewige“ übersetzt: „Das ,Ich der Ewige‘, dies Ich, mit dem als dem großen, die eigene Verborgenheit verneinenden Nein des verborgenen Gottes die Offenbarung anhebt, begleitet sie durch alle einzelnen Gebote hindurch.“ Das heißt: Dieses göttliche „Ich“ drückt jedem göttlichen Gebot ein Siegel auf, das es als Gebot der Liebe kennzeichnet, denn nur das Liebesgebot ist nach Rosenzweigs grammatikalischer Analyse reines Gebot, reine Gegenwart, unmittelbare Gegenwärtigkeit und Einheit von Bewusstsein, Ausdruck und Erfüllungserwartung, alles andere könnte auch Gesetz sein. ${ }^{52}$

So weit zum Kontext des ersten Erscheinens des Ausdrucks „der Ewige“ als Wiedergabe des Gottesnamens im Stern. Bei dem bisherigen Befund erscheint allerdings die spezifische Bedeutung des Gottesnamens als des „Ewigen“ noch nicht sinntragend für die Argumentation. Sie hätte anscheinend genauso geführt werden können mit einem „Ich der/dein HERR“ o. ä. als „Siegel der Offenbarung “ und Lautwerden des Ich.

Dennoch möchte ich behaupten, dass „der Ewige“ im Stern selbstverständlich transparent auf seine Bedeutung ist und daher sinntragend.

Daher ist zunächst nach eben dieser Bedeutung zu fragen, also was mit „Ewigkeit“ im Stern gemeint ist. Auch wenn in Teil I des Werks dem „freien Nein“ Gottes alle Ewigkeit „bloß ,andres', [...] bloß unendliche Zeit“ ist, ${ }^{53}$ wird an dem „bloß“ an dieser Stelle schon deutlich, dass Rosenzweig im Stern einen anderen Ewigkeitsbegriff als die traditionelle Vorstellung einer unendlichen

Man beachte dazu auch die Zeichnung von Anna Elisabeth Bauer (Rosenzweigs Sprachdenken, 281). Bauer stellt allerdings Gott „auf den Kopf“ und vertauscht trotz ansonsten sorgfältiger Rekonstruktion deswegen die beiden unteren Ecken des Stern.

49 Vgl. Stéphane Mosès, System und Offenbarung. Die Philosophie Franz Rosenzweigs (München: Fink, 1985), 95.

50 Rosenzweig, Stern der Erlösung, 193.

51 Dober, Die Zeit ernst nehmen, 197.

52 Rosenzweig, Stern der Erlösung, 197.

53 Rosenzweig, Stern der Erlösung, 32. 
Zeitspanne benutzt. ${ }^{54}$ Er definiert diesen am Ende von Teil II so: „Ewigkeit ist nicht eine sehr lange Zeit, sondern ein Morgen, das ebensogut Heute sein könnte. Ewigkeit ist eine Zukunft, die, ohne aufzuhören Zukunft zu sein, dennoch gegenwärtig ist. Ewigkeit ist ein Heute, das aber sich bewußt ist, mehr als Heute zu sein.“55 Ganz kurz gefasst bedeutet Ewigkeit „nicht ,sehr lange“, sondern ,heute schon““;56 etwas vorgreifend ergänze man: allerdings nur dann, wenn ihr „auf seine Stimme hören werdet“ (Ps 95,7) und auf Gottes Liebe mit eurer Liebe antwortet. ${ }^{57}$

Dieser Zusammenklang der Zeiten erklärt sich nun direkt aus der „Dialektik“ des Stern, ${ }^{58}$ wodurch auch die Brückenfunktion des Ewigkeitsgedankens deutlich wird. Dies lässt sich auch im konkreten Arbeitsprozess Rosenzweigs an diesem Werk nachvollziehen: Schon neun Tage nach Beginn der Arbeiten am Stern steht das Gerüst des Inhaltsverzeichnisses, in dem der Ewigkeitsgedanke prominent vorkommt.59 Die zeitlichen Adjektive „immerwährend“, „allzeiterneuert“ und „ewig“ sind dort bereits dieselben wie dann im endgültigen Inhaltsverzeichnis, wie es hier zur Erinnerung wiedergegeben wird: 60

\section{ERster TeIl. Die Elemente oder DIE IMMERWÄHRENDE VoRWelt Einleitung: Über die Möglichkeit, das All zu erkennen Erstes Buch: Gott und sein Sein oder Metaphysik Zweites Buch: Die Welt und ihr Sinn oder Metalogik Drittes Buch: Der Mensch und sein Selbst oder Metaethik Übergang \\ Zweiter Teil. Die Bahn oder die allzeiterneuerte Welt Einleitung: Über die Möglichkeit[,] das Wunder zu erleben}

54 Eine Diskussion der möglichen Verständnisse von „Ewigkeit“ findet sich bei Michael Theunissen, Negative Theologie der Zeit (Frankfurt am Main: Suhrkamp, 1991), 89-130.

55 Rosenzweig, Stern der Erlösung, 250.

56 Rosenzweig, Stern der Erlösung, 261.

57 Rosenzweig, Stern der Erlösung, 198.

58 Bei dem die „Synthese“ These und Antithese ja nicht aufhebt, vgl. dazu das Folgende. Es ist hier eher an den Gebrauch des Dialektischen in der Phänomenologie des Geistes (als „Wissenschaft der Erfahrung des Bewusstseins“) zu denken, der „Bibel der Philosophie“ unter den Freunden, und das so, dass Rosenzweig diese Dialektik in seinem „neuen Denken“ einer Relektüre als Geschehen von Zeit unterzieht. Diesen Hinweis verdanke ich Heinz-Jürgen Görtz; vgl. Heinz-Jürgen Görtz, Tod und Erfahrung. Rosenzweigs „erfahrende Philosophie“ und Hegels „Wissenschaft der Erfahrung des Bewußtseins“ (Düsseldorf: Patmos, 1984).

59 Rosenzweig, Brief an Margrit Rosenstock vom 31.8.1918.

60 Rosenzweig, Stern der Erlösung, vii; kursiv vom Vf. 
Erstes Buch: Schöpfung oder der immerwährende Grund der Dinge Zweites Buch: Offenbarung oder die allzeiterneuerte Geburt der Seele

Drittes Buch: Erlösung oder die ewige Zukunft des Reichs Schwelle

Dritter Teil. Die GeStalt oder Die EWIGE ÜBerWelt Einleitung: Über die Möglichkeit, das Reich zu erbeten

Erstes Buch: Das Feuer oder das ewige Leben Zweites Buch: Die Strahlen oder der ewige Weg Drittes Buch: Der Stern oder die ewige Wahrheit Tor

Ergebnis der Betrachtung: Gegenüber der immerwährenden Vorwelt und der allzeiterneuerten (Um-/Mit-)Welt ${ }^{61}$ bietet der Begriff der ewigen Überwelt die „Synthesis“62, die Immerwährendes und Allzeiterneuertes einschließt. Gegen Hegel, bei dem Ewigkeit nur absolute Gegenwart ist und nicht sein wird, „noch war sie“, ${ }^{63}$ ist eben auch der Begriff der Synthese bei Rosenzweig anders zu verstehen, nämlich als „Zeichen des Prozesses, der zwischen den im Ja und Nein Entstandenen die fertige Gestalt wachsen läßt“, und in diesem Sinne „etwas ganz andres als die idealistische ,Synthesis““. ${ }^{64}$ Gott ist im Stern das große „Und“ in der „Dialektik“ des Stern, gewissermaßen „unsere Synthesis“, „Schlußstein des übrigens auf eigenen Pfeilern errichteten Gewölbes“. 65

Vor diesem Hintergrund ist jedenfalls „der Ewige“ tatsächlich die einzig passende Übersetzung des Gottesnamens im Stern, denn der Ewigkeitsbegriff vereint für Rosenzweig ${ }^{66}$ die Aspekte des Immerwährenden und Allzeiterneuerten zugleich, nur er kann die Brücke, oder den „Schlußstein“, wie Rosenzweig sagt, bilden. Kurzgefasst und vereinfacht ausgedrückt formuliert Rosenzweig den Ewigkeitsgedanken in einem Brief an Margrit Rosenstock: ,ganz stark und si-

61 Rosenzweig, Brief an Margrit Rosenstock vom 19.11.1918.

62 Rosenzweig, Stern der Erlösung, 256.

63 Georg Wilhelm Friedrich Hegel, Enzyklopädie der philosophischen Wissenschaften im Grundrisse (1830). Zweiter Teil. Die Naturphilosophie mit den mündlichen Zusätzen (Frankfurt am Main: Suhrkamp, 1970), 50. Dazu Schindler, Zeit, Geschichte, Ewigkeit, 44-46.

64 Rosenzweig, Stern der Erlösung, 256.

65 Rosenzweig, Stern der Erlösung, 256. Die fertige Gestalt ist in diesem Fall die Erlösung Gottes selbst (Rosenzweig, Stern der Erlösung, 257). Hierzu auch ausführlicher im Folgenden.

66 Jedenfalls zum Zeitpunkt der Abfassung des Stern. Im bekannten Brief an Martin Goldner beispielsweise klingt dies nur noch schwach an, hier wird Ewigkeit in der Regel dem Immerwährenden oder Absoluten gleichgeordnet; vgl. Franz Rosenzweig, Brief an Martin Goldner (23.6.1927), in: Buber/Rosenzweig, Die Schrift, 332-338, hier 335-336. 
cher und jeden Augenblick da sein [...], wenn man es braucht - und das heisst ja ,ewig'.“67

\section{Der Gedanke der Verewigung}

Die Ewigkeit überbrückt beide Pfeiler der Systematik, sie macht in Rosenzweigs Worten ,eben den Augenblick zum Immerwährenden; sie ist die Ver-ewigung“, 68 wie er es auch nennt. Diesem Gedanken der Verewigung werden wir nun noch etwas nachspüren. Dies soll im Beantworten der Frage geschehen, wieso die Ewigkeit schon im Offenbarungsbuch des Stern eine Rolle spielt. „Der Ewige“ ist zwar in der Tat die Wiedergabe des offenbarten Gottesnamens. Doch das Adjektiv „ewig“ taucht in Rosenzweigs oben abgebildetem Inhaltsverzeichnis erst in II/3 und dann durchgehend in Buch III, also von den drei Dimensionen des Stern Schöpfung - Offenbarung - Erlösung erst in Bezug auf letztere, die Erlösung, auf, nicht schon bei der Offenbarung im eigentlichen Sinne.

Der Grund nun, warum bereits in der Offenbarung Ewigkeit eine Rolle spielt, ist das Werden Gottes. Dem Gott, der sich in seiner Offenbarung als der Werdende vorgestellt hat (אהיה אשר אהיה,", Ex 3,14) ist „die Schöpfung der Welt das Schöpferwerden, die Offenbarung das Offenbarwerden, die Erlösung das Erlöserwerden. So wird er bis zum Ende. Alles was geschieht, ist an ihm Werden."69 Jenes Werden Gottes ist „für ihn kein Sichverändern, kein Wachsen, kein Zunehmen, sondern er ist von Anfang an und ist in jedem Augenblick und ist immer im Kommen; und nur wegen dieses Zugleichs seines Immerwährend-, Allzeit- und Ewigseins muß man das Ganze als ein Werden bezeichnen. "70 Man beachte dabei die Verwendung aller drei temporalen Kategorien aus der Gliederung des Stern.

Jede Vorstellung eines zeitlichen Werdens dagegen, „wie sie eine freche Mystik und ein hochtrabender Unglaube ihm gern andichten“, prallt „,von seiner Ewigkeit ab“, 71 „,[d]enn für Gott ist die Zukunft keine Vorwegnahme; er ist ewig und der einzige Ewige, der Ewige schlechtweg; ,Ich bin' ist in seinem Munde wie ,Ich werde sein' und findet erst darin seine Erklärung. "72 In der Offenbarung des Gottesnamens scheint also bereits Ewigkeit auf.

67 Rosenzweig, Brief an Margrit Rosenstock vom 26.12.1919.

68 Rosenzweig, Stern der Erlösung, 288.

69 Rosenzweig, Stern der Erlösung, 287.

70 Rosenzweig, Stern der Erlösung, 288.

71 Rosenzweig, Stern der Erlösung, 303.

72 Rosenzweig, Stern der Erlösung, 303. Kursiv vom Vf. 
Was bedeutet nun Verewigung? Auch in der Offenbarung der Liebe Gottes und der damit verbundenen Erweckung des Selbst zur Seele geschieht bereits Erlösung, ein „Und“. Es ist noch nicht das große „Und“ der Endgestalt des Stern, sondern das „Und“, das die beiden Pole der Offenbarung, Gott und Mensch, verbindet; also gerade jene Verbindung, die in unserem Zitat aus der „Urzelle“ besonders thematisiert worden ist. In Bezug auf diesen Prozess schreibt Rosenzweig:

„Was dem Liebenden [d.h. hier Gott; A. L.] ein immer zu erneuernder Augenblick ist, das Geliebte weiß es als ewig, immer und ewig. ,Immer` ist das Wort, das über seiner Liebe geschrieben steht. Sie ist nie größer als im Augenblick, wo sie erweckt wird; sie kann nicht mehr wachsen, aber sie kann auch nicht abnehmen, sie kann höchstens sterben: das Geliebte ist treu.“73

Das bedeutet: Das Geliebte erst verewigt die Liebe Gottes und, so unerhört das zunächst klingt, macht diesen damit zum Ewigen. Das ist mit „Verewigung“ gemeint, und genau dies behauptet Rosenzweig mit Verweis auf die Kabbala:

„Der Glaube der Seele bezeugt in seiner Treue die Liebe Gottes und gibt ihr dauerndes Sein. Wenn ihr mich bezeugt, so bin ich Gott, und sonst nicht - so läßt der Meister der Kabbalah den Gott der Liebe sprechen. ${ }^{74}$ Der Liebende, der sich in der Liebe preisgibt, wird in der Treue der Geliebten aufs neue geschaffen und nun auf immer. Das ,auf ewig‘, das die Seele im ersten Überschauertwerden von der Liebe des Liebenden in sich vernimmt, ist keine Selbsttäuschung, bleibt nicht in ihrem Innern; es erweist sich als eine lebendige, schöpferische Kraft, indem es die Liebe des Liebenden selber dem Augenblick entreißt und sie einfürallemal - verewigt."75

Erst im Moment der Offenbarung an den Menschen wird Gott also der Ewige. Man könnte meinen, er würde erst dann so bekannt und genannt. Doch das wäre eine Verwechslung des im Stern vorherrschenden Ewigkeitsbegriffs mit dem traditionelleren Ewigkeitsverständnis, was allein dem immerwährenden As-

73 Rosenzweig, Stern der Erlösung, 188.

74 Pesikta XII,6: „Es heisst Jes. 43,12: „Ich verkündigte und rettete und that es kund, und kein Fremder war unter euch, und ihr seid meine Zeugen, spricht der Ewige und ich bin Gott.' ,Ich verkündigte“ in Aegypten s. Ex. 4,28: ,Und Mose verkündigte dem Volke alle Worte des Ewigen; , und ich rettete‘ am Meere, denn es heisst Ex. 14,29: ,Und die Kinder Israels gingen im Trocknen' u. s. w.; ferner das. V. 30: ,Und so rettete der Ewige. 'Und ich that kund“ am Sinai s. Ps. 76,9: ,Vom Himmel liessest du Gericht erschallen, ‘, und es ist kein Fremder unter euch`d. i. Jethro, ,und ihr seid meine Zeugen, spricht der Ewige, dass ich Gott bin.' R. Simeon ben Jochai hat gelehrt (hat diese Stelle dahin erklärt): Wenn ihr meine Zeugen seid, bin ich der Ewige, wenn ihr aber nicht meine Zeugen seid, so bin ich, wenn man so sagen darf, nicht der Ewige.“ August Wünsche, Pesikta des Rab Kahana (Leipzig: Schulze, 1885), 131-132.

75 Rosenzweig, Stern der Erlösung, 191. 
pekt nahekäme. ${ }^{76}$ Als nur Immerwährender ist Gott für Rosenzweig aber noch der verborgene Gott der heidnischen Vorwelt. Nur als ebenso allzeit Gegenwärtiger ist er in Wahrheit der Ewige, die Bezeugung setzt ja die erfahrene Gegenwart voraus.

Nun hat auch die Ewigkeit ihre Eigenzeit, die Zukunft. „[...] zur Zukunft gehört vor allem das Vorwegnehmen, dies, daß das Ende jeden Augenblick erwartet werden muß. Erst dadurch wird sie zur Zeit der Ewigkeit.“77 „Dieser Gedanke der Zukunft nun, dies daß das Reich ,mitten unter euch'ist, daß es ,heute، kommt, diese Verewigung des Augenblicks, “78 ist Rosenzweig wesentlich wichtig. Wir erinnern uns: Heute, wenn ihr seine Stimme hören werdet, tritt der gegenwärtige Augenblick in die Ewigkeit ein. Damit erweist sich der Gedanke der Verewigung in gewissem Sinn auch als Ausdruck des Prozesses zwischen Mensch und Welt und damit der Erlösung, die auch diejenige Gottes darstellt.

So demonstriert der Gedanke der Verewigung einmal mehr die zentrale Funktion des Ewigkeitsgedankens, der die „Synthese“ aus Immerwährendem und Allzeiterneuertem bildet. Insofern er ewige Gegenwart, ewiges Dasein darstellt, bildet er auch eine Brücke zu der späteren in der Verdeutschung mit Martin Buber gefundenen Wiedergabe des Gottesnamens. In einem Brief an Gertrud Oppenheim deutet sich dies schon an: „[...] wie Raschi die Offenbarung am Horeb ,Ich bin der ich bin“ umdeutet: das heißt: ich bin mit euch wie ich mit euren Vätern war. - Das bloße Sein geht eben den Menschen weiter nichts an. "79 Es ist sicher nicht falsch, hier einen Anklang an das „Ich werde da sein, als der ich da sein werde“ der Verdeutschung zu hören. ${ }^{80}$ Wie gezeigt worden ist, hat „ewig“ zur Zeit der Abfassung des Stern für Rosenzweig nicht nur, aber eben auch diese providenzielle Bedeutung. So ist wie für Mendelssohn offensichtlich auch für Rosenzweig ,in ,der Ewige“ [...] der Gott des betenden Anrufs mit-

76 Auch wenn Rosenzweig seine dynamische Ewigkeitskonzeption bereits vor dem Verfassen des Stern in einem Brief an Margrit Rosenstock entfaltet. Rosenzweig, Brief an Margrit Rosenstock vom 14.3.1918.

77 Rosenzweig, Stern der Erlösung, 252.

78 Rosenzweig, Stern der Erlösung, 253.

79 Rosenzweig, Briefe und Tagebücher 1, 426. Kursiv vom Vf. Hieran interessant ist zweierlei: dass Rosenzweig von „Ich bin der ich bin“ als ursprünglicher Bedeutung und damit als korrekter Übersetzung zunächst ausgeht, sich dennoch aber in der Interpretation Raschi anschließt und damit bereits beginnt, sich von seiner anfänglichen Interpretation zu distanzieren.

80 Kursiv vom Vf. Rosenzweig schreibt in den Arbeitspapieren zur Begründung dieser Übersetzung und zur Abgrenzung von der der Septuaginta: „Die Worte sind nicht „Philosophie der Bibel', sondern ganz aus dem Augenblick herauswachsend und dadurch freilich auch die Ewigkeit umfassend." Franz Rosenzweig, GS Bd.4, Sprachdenken im Übersetzen, 2. Band: Arbeitspapiere zur Verdeutschung der Schrift, hrsg. von Rachel Bat-Adam (Dordrecht/Boston/Lancaster: Nijhoff, 1984), 93. 
gemeint, mitgenannt“ ${ }^{81}$ Auch deswegen ist „der Ewige“ tatsächlich die passende Übersetzung des Gottesnamens im Stern - mit dem essenziellen Gedanken, dass diese Ewigkeit im Zwiegespräch mit dem erwachenden Menschen erst gewonnen werden muss. Immerwährend war Gott immer schon, aber eben nicht ewig, allezeit gegenwärtig im Sinne des Stern.

Die Übersetzung des Gottesnamens als „der Ewige“ mit diesem als „Synthese“ interpretierten Ewigkeitsverständnis im Hintergrund ist deswegen so zentral, weil es auf der temporalen Ebene Rosenzweigs Tatsächlichkeitsgedanken ausdrückt. „Der ganze geht ja wirklich nur um den einen Begriff der Tatsächlichkeit. Die Tatsache, das factum erst befreit einen von der blossen visio, zu deutsch i $\delta \varepsilon \alpha$ - Idee“ ${ }^{82}$ also vom Idealismus, den Rosenzweig zu überwinden suchte. „Nicht die Sache, nicht die Tat, erst die Tatsache ist sicher vor dem Rückfall ins Nichts“, 83 also nicht allein die Bejahung oder Verneinung, sondern das Und, wie es in der Gesamtarchitektonik des Stern nun eben der Ewigkeitsbegriff als „Synthese“ ausdrückt.

\section{Ewiges Leben und ewiger Weg}

Am offensichtlichsten erscheint der Ewigkeitsgedanke in Teil III des Stern. In ihm geht es um die Gestalt oder die ewige Überwelt, verdeutlicht an Judentum und Christentum als ewigem Leben bzw. ewigem Weg. ${ }^{84}$ Hinter dieser Aufteilung steht Rosenzweigs persönliche Antwort auf die Versuchung des Leipziger Nachtgesprächs, wie er es in dem zitierten Brief an Rudolf Ehrenberg mit Anspielung auf Joh 14,6 („Ich bin der Weg, und die Wahrheit und das Leben; niemand kommt zum Vater denn durch mich“, Luther 1912) zum Ausdruck bringt: „Es kommt niemand zum Vater - anders aber wenn einer nicht mehr zum Vater $\mathrm{zu}$ kommen braucht, weil er schon bei ihm ist“",85 wie es das ewige Leben des Judentums ausdrückt.

In der gemeinsamen Einleitung dieses dritten Teils geht es um das Gebet des Gläubigen, in dem sich das „rechte Gebet“ vollzieht, welches die Ewigkeit zum Nächsten, zum Heute macht und dadurch das Kommen des Reiches ins

81 Rosenzweig, Der Ewige, 102.

82 Rosenzweig, Brief an Margrit Rosenstock vom 13.11.1918.

83 Rosenzweig, Stern der Erlösung, 270.

84 Mit dieser Aufteilung setzt sich Rosenzweig von der verbreiteten Darstellung des Juden als ewig unterwegs seiendem Ahasver ab. Das Judentum ist bei ihm bereits am Ziel, während es das Wesen des Christentums ist, auf dem Weg zu sein.

85 Rosenzweig, Briefe und Tagebücher 1, 135. 
Heute beschleunigt. Der Christ denkt dabei sicher ans Vaterunser, und in der jüdischen Tradition gibt es viele zentrale Gebete, die diese Bitte enthalten. ${ }^{86}$

Ewigkeit ist für Rosenzweig ja gerade dadurch bestimmt, dass sie heute schon kommen kann. ${ }^{87}$ Sie kann im Heute aber nur so ihre Unvergänglichkeit bewahren, dass sie ständig wiederkommt, in der Stunde der Zeitordnung, die aber erst durch den Festkreis gestiftet wird. ${ }^{88}$ „In der alltäglich-allwöchentlich-alljährlichen Wiederholung der Kreise des kultischen Gebets macht der Glaube den Augenblick zur ,Stunde،, die Zeit aufnahmebereit für die Ewigkeit." ${ }^{89}$ Und an wen anders als „den Ewigen“ sollte dieses Gebet gerichtet sein? Ohne es zu merken, nimmt hier Rosenzweig im Grunde die Tatsache vorweg, dass „der Ewige“ auch in christlichen Bibelübersetzungen im Gebrauch ist, wie er selbst allerdings erst später bei der Ausarbeitung des gleichnamigen Aufsatzes feststellt. ${ }^{90}$

Judentum und Christentum transzendiert dann auch der dritte Abschnitt des dritten Teils, vom Stern oder der ewigen Wahrheit. Auch hier spielt Namenstheologie eine Rolle, wie ebenfalls im Brief an Rudolf Ehrenberg ausgedrückt. Rosenzweig erkennt in 1 . Kor 15,28 $8^{91}$ das große Gesprächsangebot des Christentums gegenüber dem Judentum. ${ }^{92}$

Dabei bleibt der Stern der Erlösung ein durch und durch jüdisches Buch. Bereits im Anfang von III/2 gibt Rosenzweig ja Maimonides' Ansicht über die Notwendigkeit des christlichen Wahns wieder. ${ }^{93}$ Das bedeutet biografisch auch: Der Stern als ewige Wahrheit übertrumpft ebenso Rosenstocks Kreuz der Wirklichkeit. ${ }^{94}$ Wie immer gelingt es Rosenzweig, diese Apologetik in die schönsten Worte zu kleiden:

86 Vgl. die Aufzählung zu S. 326 im Anhang des Stern, Rosenzweig, Stern der Erlösung, 480.

87 Rosenzweig, Stern der Erlösung, 321.

88 Rosenzweig, Stern der Erlösung, 323.

89 Rosenzweig, Stern der Erlösung, 324.

90 Rosenzweig, Briefe und Tagebücher 2, 1220.

91 „Wenn aber alles ihm untertan sein wird, alsdann wird auch der Sohn selbst untertan sein dem, der ihm alles untergetan hat, auf daß Gott sei alles in allen.“ (Luther 1912)

92 In seinem Brief vom 1.11.1913 an Rudolf Ehrenberg schließt er dem seinerseits ein jüdisches Gesprächsangebot an: „An diesem Punkt, wo Christus aufhört der Herr zu sein, hört Israel auf erwählt zu sein; an diesem Tage verliert Gott den Namen, mit dem ihn allein Israel anruft; Gott ist dann nicht mehr ,sein“ Gott." Rosenzweig, Briefe und Tagebücher 1, 135.

93 „Sondern es ist eben einfach ein Christlicher Verlag und weiter nichts. Und da gehört ein jüdisches Buch, ein Buch worin das Christentum von oben betrachtet wird, ein Buch worin das Christentum ein ,Wahn' genannt wird, nicht hinein.“ Rosenzweig, Brief an Eugen Rosenstock vom 27.6.1919, The Gritli Letters (Gritli Briefe), http://argobooks.org/gritli/index.html [26.11.2014].

94 Hartwig Wiedebach (Hg.), „Kreuz der Wirklichkeit“ und „Stern der Erlösung“. Die GlaubensMetaphysik von Eugen Rosenstock-Huessy und Franz Rosenzweig (Freiburg im Breisgau/München: Alber, 2010), 10. 
„Wo alles brennt, da gibt es keine Strahlen mehr. Da ist alles ein Licht. Da wird die Erde voll sein von Erkenntnis des Ewigen, wie Wasser das Meer bedecken. In diesem Meer des Lichts ist aller Weg versunken wie ein Wahn. Du aber, Gott, bist Wahrheit.“95

\title{
Am Ende das Schweigen
}

Aber Rosenzweig bleibt dabei nicht stehen. Am Ende heißt es: „Aller Name schwindet."96 Die Erlösung als Gottes großer Sabbat erlöst ihn von allem außer ihm, der Arbeit an der Schöpfung und der Not um die liebe Seele. Gott wird Einer, und dies ist in der originellen Rosenzweig'schen Schriftinterpretation dann auch sein Name: „Einer“. Dieses endzeitliche Motiv der hebräischen Bibel (Sach 14,9) gilt es noch systematisch einzulösen. „Die Erlösung erlöst Gott, indem sie ihn von seinem offenbarten Namen löst.“97 Während seit der Offenbarung alles im Namen und um des Namens willen geschieht und so zur Erlösung beiträgt, ist das Ende namenlos, über allen Namen. „Jenseits des Worts und was ist der Name andres als das ganze gesammelte Wort - jenseits des Worts leuchtet das Schweigen. Wo dem einen Namen keine andern Namen mehr sich entgegenwerfen, wo der eine Name all-ein ist und alles Geschaffene ihn und nur ihn kennt und bekennt, da ist die Tat der Heiligung zur Ruhe gekommen. [...] Wo alles heilig ist, da ist Heiliges selbst nicht mehr heilig, da ist es einfach da." 98

Interessanterweise bricht Rosenzweig an dieser Stelle mit der Wiedergabe des Gottesnamens durch „der Ewige“, auch wenn er immer noch auf diese Sinndimension anspielt:

\begin{abstract}
„Um unsrer Ewigkeit willen müssen wir das Schweigen, in das er einst und wir mit ihm versinken, vorwegnehmen und für den Namen selber das einsetzen, was er ist, solange er noch als Namen gegen andre Namen, als Schöpfer einer Welt von Sein, als Offenbarer einer Sprache von Seelen genannt wird: Herr. Statt seines Namens nennen wir ihn Herr." “99
\end{abstract}

Interessant ist die Tatsache, dass an dieser Stelle „Herr“ ohne Possessivpronomen verwendet wird, ein Gebrauch, den Rosenzweig in dem Aufsatz „Der

95 Rosenzweig, Stern der Erlösung, 422.

96 Rosenzweig, Stern der Erlösung, 265. Vgl. auch Franz Rosenzweig, „Die Wissenschaft von Gott“, in: GS Bd. 3, Zweistromland, 619-642.

97 Rosenzweig, Stern der Erlösung, 426.

98 Rosenzweig, Stern der Erlösung, 426.

99 Rosenzweig, Stern der Erlösung, 427. Kursiv vom Vf. 
Ewige“100 und, wie wir gesehen haben, bereits in früheren Briefen kritisiert. Das „[...] nennen wir ihn Herr“ im obigen Zitat weist darauf hin, dass Rosenzweig vermutlich ganz traditionell-jüdisch „Adonaj“ dachte, ${ }^{101}$ möglicherweise ohne auf den Selbstwiderspruch aufmerksam zu werden.

Man könnte aber auch darüber nachdenken, warum Rosenzweig das ganze Geschehen in Teil II des Stern als „Welttag des Herrn“ bezeichnet, wie er es im Ausblick des Übergangs von I auf II nennt ${ }^{102}$ und davon im Ausblick der „Schwelle“ von II auf III den „Gottestag der Ewigkeit“ unterscheidet. ${ }^{103}$ Gegen Hegels zeitloses „Schauen“ des Selbstbewusstseins zielt der Stern auf ein geschichtlich verortetes Schauen „am ,Ende der Tage“ des ,Welttags des Herrn“, an welchem Ende dieser in den ,Gottestag der Ewigkeit“ eintritt“. ${ }^{104}$ „Rosenzweig will mit dieser Unterscheidung der Tatsache Rechnung tragen, dass der Welt und dem Menschen Schöpfung, Offenbarung und Erlösung nicht das gleiche bedeuten, was sie Gott bedeuten. "105 Auf der Ebene der Gottesbezeichnung scheint somit jedoch „der Ewige“ eine verheißungsvollere Zukunft zu haben als „der Herr“, wogegen die zuletzt zitierte Textstelle des Stern doch das Gegenteil nahelegte. ${ }^{106}$

Die Verwendung von „Herr“ für den Gottesnamen setzt sich jedenfalls in Rosenzweigs Übersetzung der Hymnen von Jehuda Ha-Levi fort. ${ }^{107}$ Könnte dies - neben der Reverenz an Luther natürlich - erklären, warum die Verdeutschung mit „Herr“ als Versuch beginnt? ${ }^{108}$ Dann könnte Rosenzweigs Zugeständnis im Aufsatz „Der Ewige“, wo der „Herr“ mit Possessivpronomen im Sinne von „Adonaj“ als Wiedergabe des Gottesnamens gelobt wird, gewissermaßen auch eine Selbstrechtfertigung für diese Übersetzungsphase sein. Jedenfalls versteht man die Sympathie, die Rosenzweig dafür äußert, besser, wenn man wahrnimmt,

100 Rosenzweig, Der Ewige, 99.

101 Vgl. dazu die im Anhang des Stern vermerkte Stelle aus der rabbinischen Tradition (Pes. 50a zu Ex 15,3). Rosenzweig, Stern der Erlösung, 486. Auch in III/1 des Stern spricht Rosenzweig - an einer systematisch durchaus zentralen Stelle - von „Gott der Herr“ als dem jüdischen Gott, ja am Ende der „Schwelle“ allgemein davon, dass wir in der erlösten Überwelt „Gott, erlöst von aller Arbeit des Sechstagewerks und aller lieben Not um unsere arme Seele, als den Herrn“ schauen. Rosenzweig, Stern der Erlösung, 340, 291.

102 Rosenzweig, Stern der Erlösung, 95-99.

103 Rosenzweig, Stern der Erlösung, 288-291.

104 Görtz, Tod und Erfahrung, 324.

105 Hans-Jürgen Görtz, In der Spur des „neuen Denkens“. Theologie und Philosophie bei Franz Rosenzweig (Freiburg im Breisgau: Alber, 2008), 170.

106 Rosenzweig, Stern der Erlösung, 427.

107 Schmahl, Tetragramm, 122-128.

108 Rosenzweig, Brief an Martin Goldner (23.6.1927), 333. 
dass er auch selbst „Herr“ für den Gottesnamen verwendet hat, punktuell auch im Stern.

Der Bruch mit der Wiedergabe des Gottesnamens durch „der Ewige“ und die Rückbesinnung auf eine traditionellere Wendung verlässt den Bannkreis des Stern und wird wirksam in der sich anschließenden eigenen Übersetzungsarbeit, wo Rosenzweig durchweg andere Wiedergabemöglichkeiten als „der Ewige“ bemüht. Der Gebrauch des Personalpronomens in Kapitälchen in der Verdeutschung ist nur das prominenteste Ergebnis dieses erneuten Ringens. ${ }^{109}$ Wir haben gesehen, dass der Ewigkeitsgedanke, wie er im Stern gedacht worden ist, dabei dennoch weiterhin inhaltlich nachklingt. Auch auf Martin Buber hat er seine Wirkung gehabt. ${ }^{110}$

\section{Fazit}

Die Offenbarung des Gottesnamens ist bekanntermaßen Herzstück des Stern. Rosenzweig wendet in der Konstruktion des Stern nun diese Überlegung von der zentralen Bedeutung des Namens auf die ihm überlieferte Wiedergabe des Gottesnamens durch „der Ewige“ an, sodass der Ewigkeitsgedanke die „Synthese“ in der Systematik des Stern darstellt. Damit ist das Ewigkeitsverständnis des Stern ein reicheres als das traditionelle Ewigkeitsverständnis nur einer langen Zeitspanne. Es umfasst sowohl den traditionellen Aspekt des Immerwährenden als auch den der allzeiterneuerten Gegenwart. Die Gegenwartsbedeutung des Gottesnamens, wie sie im Prozess der Verdeutschung mit Martin Buber herausgearbeitet wird, ist im Stern der Erlösung somit bereits vorgedacht. Doch selbst wenn „der Ewige“ auch im Stern bereits Gott der lebendigen Anrede ist, geht die Verdeutschung noch einen Schritt weiter. „Der Ewige“ oder „der Herr“ sind „ja doch eben nur Sinn, und nicht, was beim Tetragramm das Wesentliche ist, sinnträchtiger Name“. ${ }^{111}$ Wenn die Verwendung des Ausdrucks „der Ewige“ als Wiedergabe des Gottesnamens im Stern in einer gewissen Spannung zur manchmal verwendeten Wiedergabe durch „Herr“ steht, so hatte Rosenzweig an den

109 Zur Genese dieses Verdeutschungsansatzes siehe Andreas Losch, „Kann Gott einen Namen haben? Martin Buber befragt die jüdische Geistes- und die Religionsgeschichte. Zu ungedruckten Typoskripten Bubers“, in: Daniel Krochmalnik/Hans-Joachim Werner (Hg.), 50 Jahre Martin Buber Bibel. Internationales Symposium der Hochschule für Jüdische Studien Heidelberg und der Martin Buber-Gesellschaft (Berlin u. a.: Lit, 2014), 165-184.

110 Vgl. Andreas Losch, „Ich, Du und ER. Franz Rosenzweigs Einfluss auf die Abfassung von Ich und Du“, in: Im Gespräch. Hefte der Martin Buber-Gesellschaft 16 (2015), 19-35.

111 Rosenzweig, Brief an Martin Goldner (23.6.1927), 336. 
entsprechenden Übersetzungsstellen, so die hier entwickelte Annahme, vermutlich den „guten Ursprung der schlechten Übersetzung Herr“ im Blick, also den Vokativ Adonaj. ${ }^{112}$

Rosenzweig wird die Missverständlichkeit des Ewigkeitsgedankens aufgrund der vorherrschenden traditionellen Bedeutung des Begriffs später wieder bewusst, sodass er ihn beispielsweise im Brief an Martin Goldner weitgehend auf das traditionelle Verständnis beschränkt. Hieraus wird verständlich, dass in der Verdeutschung mit Martin Buber eine andere Wiedergabe des Gottesnamens gefunden werden musste, die aber die inhaltliche Pointe der immerwährenden Gegenwart bewahrt. Da diese Gegenwart im jeweiligen Heute von dem „wenn ihr auf seine Stimme hören werdet“ abhängt, hat diese Gegenwart noch etwas Zukünftiges, sie ist mehr eine verheißene Möglichkeit als eine einklagbare Wirklichkeit. „Ich werde dasein, als der ich dasein werde“ drückt dies in passender Weise aus. ${ }^{113}$

112 Rosenzweig, Brief an Martin Goldner (23.6.1927), 337.

113 MBA (= Martin Buber Archiv Jerusalem) Arc. Ms. Var. 350 gimel 5, Übersetzungsvorlage, 8. Kursiv vom Vf. Es bliebe zu fragen, ob auch für Buber diese futurische Brechung nahegelegen hat. Sein erster Übersetzungsvorschlag im Rahmen des Verdeutschungsprojekts folgt zwar Luthers Futur, doch in der ersten Auflage von Ich und $\mathrm{Du}$ beispielsweise heißt es noch „Das Wort der Offenbarung ist: Ich bin der ich bin.“ (Martin Buber, Ich und Du, Leipzig: Insel, 1923, 129; kursiv vom Vf.). Ab der Sammelausgabe „Dialogisches Leben“ (Martin Buber, Dialogisches Leben, Zürich: Müller, 1947) schreibt Buber zwar „Ich bin da als der ich da bin“ (ebd., 120), doch auch hier fehlt das Futur. 
Brought to you by | Universitaetsbibliothek Kassel Authenticated

Download Date | 12/14/15 4:11 PM 